Cinémas

Revue d'études cinématographiques

Journal of Film Studies

\title{
Montrer, dire et saisir l'espace dans le cinéma des premiers temps : le cas du Great Train Robbery
}

\section{Charles Perraton et Nathalie Bouchard}

Volume 5, numéro 3, printemps 1995

Cinélekta 1

URI : https://id.erudit.org/iderudit/1001144ar

DOI : https://doi.org/10.7202/1001144ar

Aller au sommaire du numéro

Éditeur(s)

Cinémas

ISSN

1181-6945 (imprimé)

1705-6500 (numérique)

Découvrir la revue

Citer cet article

Perraton, C. \& Bouchard, N. (1995). Montrer, dire et saisir l'espace dans le cinéma des premiers temps : le cas du Great Train Robbery. Cinémas, 5(3), 9-27. https://doi.org/10.7202/1001144ar
Résumé de l'article

Les deux auteurs retracent ici, à travers une analyse serrée du film The Great Train Robbery (1903), quelles stratégies ont été déployées par le réalisateur E. S. Porter et quelles compétences ont été exigées des spectateurs dans la création d'un nouvel espace filmique. Au coeur des mouvances stylistiques traversant le cinéma des premiers temps, le film émerge du croisement de deux systèmes cinématographiques : l'esthétique de l'attraction et l'esthétique de la narration. Cette hybridité encourage une liberté d'expérimentation de la part du créateur et des spectateurs, elle ouvre à la création d'un contexte encyclopédique inédit et à la production de nouvelles règles de lecture de l'espace filmique. 


\section{Montrer, dire et saisir l'espace dans le cinéma des premiers temps: le cas du Great Train Robbery ${ }^{1}$}

\section{Charles Perraton et Nathalie Bouchard}

\section{RESUME}

Les deux auteurs retracent ici, à travers une analyse serrée du film The Great Train Robbery (1903), quelles stratégies ont été déployées par le réalisateur E. S. Porter et quelles compétences ont été exigées des spectateurs dans la création d'un nouvel espace filmique. Au cœur des mouvances stylistiques traversant le cinéma des premiers temps, le film émerge du croisement de deux systèmes cinématographiques: l'esthétique de l'attraction et l'esthétique de la narration. Cette hybridité encourage une liberté d'expérimentation de la part du créateur et des spectateurs, elle ouvre à la création d'un contexte encyclopédique inédit et à la production de nouvelles règles de lecture de l'espace filmique.

\footnotetext{
ABSTRACT

In a close analysis of the film The Great Train Robbery, the two authors reevaluate the strategies employed by director E. S. Porter as well as the skills demanded of spectators in the creation of a new filmic space. Amid the changing stylistic allegiances of cinema's first years, this film emerged at the crossroads of two cinematographic systems: the esthetic of attraction and the esthetic of narration. This hybridity encouraged a freedom of expression on the part of both filmmaker and spectator. It opened the way for the creation of a previously unknown encyclopedic context, and for the production of new rules for reading filmic space.
} 
Nous nous proposons, dans le cadre de cet article, de mettre en évidence les stratégies auxquelles a eu recours le cinéaste des premiers temps Edwin S. Porter pour introduire et susciter la saisie d'un nouvel espace filmique par son spectateur. L'auteur du Great Train Robbery oriente en effet son spectateur vers une nouvelle piste de lecture des images qui lui sont données à voir. Dès ses débuts chez Edison, Porter expérimente de nombreuses nouveautés techniques (mouvements de caméra, angles de vue, gros plan, montage, etc.) et tente dans The Life of an American Fireman et dans The Great Train Robbery, tous deux de 1903, ses premiers "scénarios" par découpage narratif. Il voulut profiter de ces deux films pour mettre à profit certaines idées sur le gros plan et sur la manière de réaliser pour raconter des histoires plus longues et plus efficaces. Il n'hésite pas à obliger son spectateur à quitter sa place confortable de "voyeur en position frontale et centrale" assis dans le "meilleur siège disponible " pour l'inviter, par exemple, à monter sur un des wagons du train afin d'assister de plus près (plan 4) à une lutte féroce entre un bandit et le chauffeur du train (fireman).

Il s'agira donc pour nous d'examiner les stratégies employées pour favoriser un type particulier de représentation de l'espace, tout en montrant comment et pourquoi The Great Train Robbery est un film qui procède simultanément de deux systèmes: l'un, que d'aucuns nomment "esthétique de l'attraction", est passablement organisé au moment où est réalisé le film; l'autre, reconnu pour relever d'une "esthétique de la narration ${ }^{2}$ ", se trouve à peine en émergence à ce moment. Il faudra en effet attendre une dizaine d'années avant que ne s'institue, grâce notamment à la contribution exceptionnelle de D. W. Griffith, un nouveau mode de représentation connu sous le nom de "mode de représentation institutionnel " $\left(\mathrm{MRI}^{3}\right)$.

Avec son Great Train Robbery, le travail de Porter se trouve pris dans une poussée déterritorialisante qui contribue à la production de nouveaux moyens capables de concrétiser le projet de raconter une histoire. Car il s'avère difficile de raconter une histoire de vol de train en s'en tenant aux règles du système des attractions sans être subordonné aux pouvoirs narratifs du bonimenteur. La situation expérimentale du cinéma des débuts, ainsi 
que les importants moyens financiers fournis par Edison (Edison Manufacturing Company) donnent l'occasion à Porter de réaliser son projet. Même s'il n'est pas le seul à explorer de nouvelles avenues cinématographiques, Porter peut être considéré comme un exemple et même un symptôme des conditions matérielles et pragmatiques nécessaires pour l'introduction d'un nouvel espace filmique. Commençons par montrer que la réalisation et la réception du Great Train Robbery participent de deux systèmes cinématographiques fondamentalement différents (divergents).

\section{La pragmatique de l'espace au cinéma}

Parce que nous considérons chaque film comme un véritable acte de langage, nous pensons utile de nous référer à la pragmatique d'Austin pour mieux comprendre le film de Porter. En effet, et c'est là son aspect locutoire, le film montre d'abord un espace dans lequel évoluent les personnages et où se produit l'action. Sous cet aspect, il renvoie aux significations de l'espace représenté. Par ailleurs et du fait même qu'il montre des images, le film veut dire quelque chose. Et sous cet aspect, la représentation qu'il propose de l'espace implicite une force illocutoire, c'est-à-dire que sa manière de représenter attribue une valeur à ce qui est montré. Puis, un espace naît des effets perlocutoires de ce qui est montré et de ce qui est projeté dans le film, ce qui renvoie à la saisie du spectateur.

Porter donne à son film une forme qu'il espère adaptée au projet de raconter $/$ montrer $^{4}$ une histoire plus longue et mieux articulée. Ainsi peut-on considérer certains plans de son film (plans 4 à 7 surtout) comme des portions d'un même espace que le spectateur en vient à se représenter comme l'univers de l'action (l'espace des personnages et de leurs relations).

À l'occasion de l'analyse de ce film, il s'agit de voir comment de tels modes de représentation se subordonnent au projet du film, à ce que le réalisateur veut dire, à ce qu'est son "Idée " " du cinéma. Nous nous demandons comment Porter parvient à organiser ce qu'il montre et à dire ce qu'il veut dire de manière à ce que le spectateur saisisse son intention. C'est donc se poser le problème de la saisie du film comme acte de compréhension et 
d'interprétation qui achève le processus de production de sens. Mais avant d'expliciter davantage ce que nous entendons par les systèmes attractif et narratif, situons d'abord le film dans son contexte.

\section{Le Great Train Robbery et son époque}

Tourné en quatre jours au mois de novembre 1903 en banlieue de New York, The Great Train Robbery constitue " [...] une fidèle reproduction des véritables hold-up commis dans le Far West par diverses bandes renommées de hors-la-loi ${ }^{6} »$. Ce film de 14 plans pousse le souci d'authenticité au point d'inclure un nombre important de figurants au plan 6, dans le but de faire vrai. Produit par la Edison Manufacturing Company, qui le vendait aux exploitants de salles de cinéma au coût de $111 \$$, il est enregistré auprès de la Library of Congress américaine le $1^{\text {er }}$ décembre de la même année. Dès sa première projection au Huber's Museum, le film obtient un succès retentissant et il devient une importante source de profit pour les studios Edison. Pendant plusieurs années, il constitue d'ailleurs la principale attraction des nickelodeons, ces salles populaires de cinéma qui ouvrent leurs portes un an et demi plus tard. Devant un tel succès, Porter lui-même et d'autres cinéastes imitèrent ou copièrent la formule; en témoignent les titres suivants: The Great Bank Robbery et The Little Train Robbery d'Edwin S. Porter, The Great Train Robbery et The Bold Bank Robbery de Sigmund Lubin, The Hold-up of the Rocky Mountain Express, Across the Great Divide (1913). Avec les années, plusieurs critiques y ont même reconnu les premiers traits du genre western au cinéma: le hold-up, la poursuite et le finale où tout le monde se tire dessus.

Le spectateur du cinéma des premiers temps differe certes de celui d'aujourd'hui quant à son statut socio-économique et au climat culturel qui façonne à la fois ses expériences, ses compétences et ses attentes. La majorité des individus participent à un ensemble de traditions populaires et de divertissements ${ }^{7}$ dans lequel puisent abondamment les cinéastes des débuts. Le cinéma s'adresse alors principalement aux humbles et petites gens de la ville et ne deviendra un divertissement bourgeois qu'une quinzaine d'années plus tard. L'encyclopédie ${ }^{8}$ du public américain de 
1903 inclut ainsi des éléments capables de lui servir de cadre de référence. Ainsi en est-il de diverses pièces de thêâtre dont l'histoire se rapproche grandement du film de Porter (pensons ici à The Great Train Robbery de Scott Marble et à la pièce de theâtre The Great Train Express Robbery, jouée par une troupe populaire en tournée rurale à cette époque), des Wild West Shows ainsi que de nombreux articles de journaux racontant des vols de trains dans l'Ouest et d'autres faits divers violents (on dénombre pas moins de 29 hold-up de trains en 1900). L'auditoire est également familier avec les films anglais à succès de 1900-1902, qui ouvrent la voie à plusieurs innovations techniques'. En examinant le contexte de réception, on constate donc que le public de l'époque participe à un esprit d'aventure et de découverte.

De plus, le public des films du début du siècle s'avère particulièrement sensible aux nouveaux dispositifs de médiation (de la lanterne magique au cinématographe) et à leurs effets sur la perception. Noël Burch parle d'une force "scientiste" (p. 34) qui traverse l'époque et qui donne au public un goût marqué pour les images qui reproduisent la vie. Ouvert, ce public accueille tout ce qui contribue à repousser les limites du visible en travaillant notamment à l'analyse et à la synthèse du mouvement.

\section{Du régime des attractions à celui de la narration}

Pour Gaudreault, l'esthétique de l'attraction ${ }^{10}$ est dominée par deux périodes de l'histoire du cinéma : celle des films à un seul plan (1895-1902) et celle des films à plusieurs plans non continus (1902-1908). Or, nous l'avons vu, cette esthétique se transforme progressivement en un système de la narration grâce à l'augmentation du nombre des plans et au perfectionnement du montage. Au cours de la période des films à un plan, les cinéastes tournent des plans qui constituent en eux-mêmes des petits récits complets et autonomes". Le Great Train Robbery a été réalisé durant cette deuxième période du système des attràctions, dans un contexte où les films sont constitués de plusieurs plans non continus. Dès 1902, en effet, le nombre des plans se multiplie (on en compte déjà une dizaine) sans que ne change véritablement la nature de chacun pris isolément. La suite des plans est faite de la simple juxtaposition de petits tableaux autonomes ${ }^{12}$. 
La période du film à plusieurs plans continus s'organise graduellement selon les règles de ce qui deviendra le MRI; le tournage est planifié et exécuté en fonction du montage. À partir de 1908, les cinéastes fractionnent leurs scènes pour les unir par le montage, le cinéma adhérant ainsi peu à peu à l'esthétique de la narration.

Dans le système des attractions, on privilégie les images pour elles-mêmes plutôt que de s'attarder aux histoires que ces dernières pourraient raconter. C'est le cas du Great Train dont le succès se trouve en grande partie redevable à ses qualités attractives. On y insiste sur le mouvement, sur les illusions (au plan 1, on voit le train en surimpression dans la fenêtre du bureau du télégraphiste; au plan 3, le paysage en surimpression dans le fourgon postal; etc.) et sur les effets spectaculaires (c'est le cas, au plan 3, de l'explosion du coffre; au plan 13, de la fumée des coups de feu; etc.). À la différence du régime narratif, tous ces effets spéciaux et ces moments spectaculaires ne sont pas subordonnés à l'histoire racontée, mais existent par eux-mêmes et cherchent à interpeller directement le spectateur. Le train constitue à cet égard un "bon sujet" à filmer à cause de sa mobilité (les plans 3, 4, 7 et 8) et de son incidence sur le paysage qui défile. Dans la mesure où il relève du système attractif, le film cherche moins à impliquer le spectateur face aux personnages ou à l'histoire mise en scène qu'à provoquer ses réactions. Il travaille plutôt à le faire passer de la surprise (face aux bandits qui surgissent dans le bureau du télégraphe) à l'étonnement (d'assister au vol des avoirs de passagers ferroviaires); de la stupéfaction (qu'un des bandits jette le chauffeur en bas du train en marche) à la frayeur (lorsque bandits et justiciers se tirent dessus ${ }^{13}$ ); de la peur (lorsque le chef des bandits tire à bout portant sur l'auditoire) à l'admiration (devant le mouvement du train); de l'émerveillement (à voir les dangereuses contrées du Far West) à l'amusement (de voir la danse des cow-boys); etc. Le Great Train Robbery comble également le désir d'exotisme de son public (plans 8, 9 et 11) en mettant en scène de lointaines "montagnes sauvages" du Far West, une "belle vallée où coule un ruisseau ${ }^{14}$ " et "[...] les mœurs rudes d'une population encore peu touchée par la civilisation ou qui l'a oubliée" (Rieupeyrout, p. 75). 
Ainsi, "[...] les cinéastes des origines considéraient (de manière plus ou moins consciente) chaque plan comme une unité autonome et autosuffisante ayant comme finalité première non pas de présenter un petit segment temporel de l'action mais, au contraire, l'intégrité de l'action qui se déroule dans un espace homogène " (Gaudreault, 1980, p. 125). Or, à partir de 1902, les films commencent à compter plus d'un plan et à utiliser le montage pour les assembler. L'aspect pluriponctuel de la composition les fait se distinguer des films à un seul plan réalisé par une caméra fixe en introduisant dans le montage des changements de points de vue. Grâce à la thématique de la coursepoursuite, les cinéastes ont élaboré une forme de montage très simple qui consiste à juxtaposer des plans joints par des raccords de proximité spatiale et temporelle, ce qui est le cas de presque tous les plans du Great Train Robbery.

Mais malgré la pluriponctualité des films, le plan reste longtemps perçu comme un tableau autonome. C'est que les cinéastes participent toujours d'un régime, dont le but premier reste celui de montrer des images plutôt que de raconter des histoires. On le retrouve évidemment dans le Great Train Robbery, dont chaque plan possède encore un titre original: "Battle to the Death" (plan 13), "Realism" (plan 14), etc. Chaque plan doit d'ailleurs être enregistré et obtenir son propre copyright pour être protégé aux États-Unis à cette époque.

Cependant, lorsque les cinéastes commencent à réunir des plans analysant un même espace, pareille conception commence à perdre du terrain. On se met peu à peu à raccorder des plans soutenant une même action et la présentant sous des angles différents. Le changement de plan donne la chance au spectateur de voir sous un meilleur angle une action qui, imperturbable, continue malgré ce changement. Par ce travail sur la temporalité, le montage permet d'accéder à des niveaux inconnus du discours et d'échapper aux contraintes de la monstration, c'est-àdire de l'espace représenté et de sa simple "re-présentation", au profit de l'espace filmique de la narration. Le perfectionnement des règles de montage, dû notamment au travail de Griffith, permit l'émergence du MRI. 
Entre les attractions et la narration, The Great Train Robbery

Même s'il participe du système des attractions, The Great Train Robbery ne s'en éloigne pas moins de bien des façons. Il semble en effet peu satisfaisant de ne l'évaluer que sur la base de ce que certains ont appelé "le mode de représentation primitif" $\left(M R P^{15}\right)$. Porter cherche à raconter des histoires, et plus particulièrement celle du vol des passagers d'un train, vol qui sera puni par des justiciers qui viendront rétablir l'ordre et la paix. En fait, il se pose le problème d'essayer de raconter une histoire qui soit spécifiquement cinématographique, sans avoir à recourir au bonimenteur. De tels efforts pour échapper aux règles du système n'étaient possibles que dans le contexte d'expérimentation qui prévalait alors. Certes, avant Porter, Georges Méliès avait déjà eu l'idée de raconter/montrer des histoires; c'est ce qu'on retrouve, par exemple, dans son Voyage dans la lune. Mais ses films restaient trick motivated et donc conçus pour raconter " [...] une histoire dans l'unique but d'enjoliver et de lier la présentation de ses derniers trucs" (Gaudreault, 1984b, p. 210). Pour sa part, Porter cherche à raconter en la montrant une histoire dans le but de proposer une conclusion en forme de leçon de morale au spectateur: que les bandits sont violents et prêts à tout pour obtenir de l'argent et qu'il ne faut pas sympathiser avec eux.

Dans The Great Train Robbery, le spectateur n'assiste pas plus à la projection d'un film à un seul plan, qu'il ne regarde un film issu du système narratif (dont l'ensemble des plans continus aurait à satisfaire les règles du MRI). Et, parce que certains de ses plans sont continus, le film échappe également à la périodetampon qu'identifie André Gaudreault, période dont les films sont faits de plusieurs plans non continus.

Plusieurs théoriciens ont fait état du fort sentiment de continuité et de fluidité qui se dégage des plans 4 à 7 , grâce à un montage qui privilégie une certaine forme de continuité se rapprochant de ce qui allait devenir au milieu des années dix le MRI. Dans le catalogue Edison, ils sont intitulés: "The Fight on the Tender", "The Train Uncoupled", "Exterior of Passenger Coaches" et "The Escape ${ }^{16}$ ".

Cette impression de "continuité et de fluidité" se dégageant de la succession de ces quatre plans provient du fait de la trans- 
gression de la règle de discontinuité entre les plans du système attractif. Les plans cessent d'être de simples petits tableaux autonomes, dans la mesure où ils forment un tout articulé fait de petits segments temporels d'une même action. Les raccords entre les plans 5, 6 et 7 présentent ainsi une contiguïté spatiale et une continuité temporelle qui s'avèrent plus évidentes ${ }^{17}$ encore entre les plans 4 et 5 . Le spectateur voit et comprend qu'il est tantôt sur le wagon postal (plan 4), tantôt en bas de la locomotive près des rails (plan 5). Ces deux plans présentent les portions d'un même espace et forment un syntagme narratif qui fait participer le film à un autre régime que celui des attractions. La tâche revient au spectateur d'émettre des hypothèses sur la nature du lien unissant ces plans et d'inférer l'existence d'un nouvel espace, sorte de "grand espace" de la narration où se déroule le vol du train. Dans cette partie du film, on voit que Porter essaie de s'affranchir du travail du bonimenteur normalement chargé de fournir les compléments narratifs d'information qui s'imposent. Ses images parlent d'elles-mêmes. Les plans 4 et 5 permettent alors de prendre temporairement congé du bonimenteur, le temps de laisser le film "montrer ce qu'il a à dire ". Mais ce congé sera de courte durée, car l'arrivée des plans 10 et 11 soulèvera le problème d'énoncer ultérieurement dans le "récit" une deuxième ligne d'action qui commençait au moment où une première qui lui était simultanée avait déjà eu lieu. De cette manière, nous pouvons dire que The Great Train Robbery participe à deux régimes en même temps (l'attraction et la narration), dans la mesure où le second système se construit à partir de certains éléments mis de l'avant lors de l'expérimentation.

Se pose alors pour nous la question de savoir comment le spectateur peut arriver à comprendre un film qui participe à deux régimes à la fois, comment il peut inférer une deuxième ligne d'action à partir des plans 10 et 11 que d'aucuns considèrent "[...] une ellipse foncièrement déroutante" (Gaudreault, 1983, p. 23) ? Comment il peut saisir l'idée du film sans être bloqué par l'incohérence du propos ${ }^{18}$ ? La saisie du spectateur dépend en fait du travail du bonimenteur, dont le commentaire en voice over est issu en droite ligne de la tradition du bonimenteur des lanternes magiques, qui restituait la narrativité des histoires racontées à 
partir d'images immobiles et déficientes. Autrement, on demande au spectateur d'émettre des hypothèses sur la nature des liens temporels unissant chaque plan. L'apparition du montage suppose ainsi un travail accru de la part du spectateur car, selon Roger Odin, toute articulation de plan à plan recèle une sorte de "consigne de lecture" qu'il faut parvenir à décoder (telle coupe est un flash-back, une ellipse, etc.), elle fait appel à des règles de cohérence tributaires de l'institution cinématographique (p. 70). La compréhension de ces consignes a dû faire l'objet d'un apprentissage complexe et graduel chez les spectateurs de cinéma.

C'est le bonimenteur qui résout les problèmes de lisibilité du film, en se transformant en véritable narrateur-suppléant. Car même si on participe toujours au régime des attractions, la multiplication des plans menace la saisie du film par le spectateur puisque " [...] chaque hiatus de caméra donne lieu à des hiatus spatiaux ou temporels, creuse des trous dans le fil narratif et laisse place à l'interprétation quand ce n'est pas à l'incompréhension" (Gaudreault, 1988, p. 167). Pour rétablir la continuité entre les plans et s'assurer que le spectateur comprenne ce qui se passe à l'écran, il n'y a donc que deux solutions possibles: confier la direction du récit à la voix narrative du bonimenteur ou encore aux intertitres (qui apparaissent la même année que le Great Train Robbery). Dès que le bonimenteur précise que les plans 10 et 11 se passent en même temps que les plans précédents, une large part des problèmes de compréhension de l'auditoire sont résolus. Le film de Porter dépend donc du bonimenteur pour faire comprendre qu'une action que l'on voit présentement à l'écran puisse avoir eu lieu auparavant. L'expérimentation de Porter n'est pas risquée pour le public de l'époque puisque la tradition de visionnement des films inclut justement le travail du bonimenteur et qu'il ajoute des éléments pour favoriser la compréhension plutôt qưil n'en retranche ${ }^{19}$.

\section{Le spectateur à l'horizon des expériences de Porter}

Placé devant un film de Porter, situé à mi-chemin entre deux systèmes cinématographiques, voyons ce que le spectateur peut en comprendre. Le spectateur de l'époque peut en fait comprendre quelque chose de nouveau, il peut comprendre que deux plans qui se suivent (les plans 4 et 5 ) ne racontent pas 
nécessairement des histoires différentes. Une telle saisie se fait au profit d'un nouveau rapport spectatoriel, dont la règle s'énoncerait ainsi : "Le tout (spatial) est supérieur à la somme de ses parties (visuelles)." Car le tout (le film avec ses quatorze plans) nous dit que les portions d'espace, d'action et de temps peuvent constituer des matériaux utiles à la construction d'une histoire, d'une narration. Ainsi, "[...] chaque tableau apporte maintenant sa "brique" à l'édifice narratif [...] et nous passons d'un décor à l'autre avec une logique inexorable" (Burch, p. 43).

Le film oblige le spectateur de l'époque à se référer au système des attractions, mais l'astreint aussi à inférer l'existence - ou plutôt l'horizon — d'un autre système (le MRI). Porter ne réalise certainement pas son film pour le subordonner à un régime qui existera beaucoup plus tard. Pourtant, il est traversé par une idée du cinéma qui lui permet de penser utile la transgression de règles et de conventions pour expérimenter. Porter se demande, et il n'est pas le seul, ce que pourrait être le cinéma s'il n'était pas exclusivement subordonné au système des attractions. Il se prend à imaginer que le cinéma puisse raconter des histoires, de manière à ce que le spectateur comprenne que quelque chose se passe spatialement et temporellement. En passant du plan $4 \mathrm{au}$ plan 5 grâce à un raccord de contiguïté spatiale et de continuité temporelle, il s'approche du MRI tout en fournissant une information supplémentaire à son spectateur. Si le bonimenteur explique que les bandits ont maîtrisé le mécanicien et que celui-ci leur détache la locomotive, il devient redondant par rapport à l'image que voit le spectateur. En travaillant à partir de ces deux régimes cinématographiques ${ }^{20}$, Porter jette comme "un virus dans le sac encyclopédique des spectateurs de l'époque". Avec son Great Train Robbery, il contribue à la création d'un nouveau contexte encyclopédique par la production de nouvelles règles de lecture ${ }^{21}$.

Pas plus que Porter ne constituait la condition nécessaire à l'émergence du MRI, le MRI n'était la seule voie conséquente pour le cinéma. Ce dernier aurait certes pu privilégier des esthétiques autres que celles de la narration et de l'attraction, ce qui inaugurait par là d'autres perspectives spectatorielles.

Même si The Great Train Robbery s'inscrit dans un mouvement déterritorialisant, il n'a pas égaré son public, au contraire. 
Les plans qu'il soustrait au système des attractions ouvrent la voie aux nouveaux modes d'inférence, car ils ne forment pas d'ellipse, grâce à leur contiguïté spatiale et à leur continuité temporelle. Le nouveau système s'autodéfinit donc en énonçant sa règle. Au cinéma, cette règle ne doit-elle pas être visible? C'est-à-dire que le spectateur ne comprend qu'à partir de ce qu'il sait déjà et/ou à partir de ce qu'il voit. Tout ce qui pourrait s'énoncer à partir de règles qu'il ne connaît pas risque de ne pas être compris, à moins que ce qui lui est montré et ce qui le rend intelligible (la règle ou la structure qui l'organise) ne soient visibles en même temps. Voilà sans doute pourquoi les changements aux règles ne peuvent être que minimaux à chaque fois. La nouvelle règle de montage s'énonce au moment de son énonciation, faisant de ces images et de leur enchaînement un cas d'application de la règle qu'il s'agit d'inférer (pour C. S. Peirce, cette inférence est abductive) : le tout est supérieur à la somme de ses parties, c'est-à-dire que les portions d'espace, d'action et de temps constituent les matériaux (des briques) nécessaires pour la construction de la narration. Dans ce sens, Porter est minimaliste puisque les changements qu'il opère introduisent de petites portions de récits. Cette attitude minimaliste lui est nécessaire pour ne pas perdre son spectateur. Pour se passer du bonimenteur (dans les plans 4 et 5), il lui a fallu en raconter peu à la fois.

The Great Train Robbery participe ainsi à un troisième régime, un régime dont les règles s'énoncent au fur et à mesure de leur application. Au sein de ce régime, les règles doivent être simples et explicites et les déplacements minimaux par rapport au système en place, pour éviter la déroute spectatorielle.

Le mérite de Porter aura été, et cela malgré l'inconfort de sa situation et le caractère paradoxal de son entreprise, d'ouvrir la voie à l'expérimentation cinématographique. Sa contribution se sera faite sous le couvert d'une idée du cinéma qui, après la période des spectacles du MRP et avant celle de la narration du MRI, permettait de raconter des histoires tout en montrant ce qui est à raconter, de le faire sans avoir besoin de tout montrer ni d'avoir toujours besoin d'un bonimenteur pour le faire.

Université du Québec à Montréal 


\section{ANNEXE \\ Plans du film The Great Train Robbery}

Plan 1 : Interior of Railroad Telegraph Office ${ }^{22}$ (1:09)

Deux bandits entrent dans le bureau du télégraphiste et le forcent à donner l'ordre d'arrêter le train; le train s'arrête et le conducteur vient à la fenêtre recevoir l'ordre de s'approvisionner en eau à cette station; dès qu'il repart, les bandits frappent et ligotent le télégraphiste.

Plan 2: The Railroad Water Tank $(0: 34)$

D'autres bandits se cachent derrière le réservoir à eau; dès que le train emmagasine l'eau et repart, les bandits y montent.

\section{Plan 3: Interior of Express Car ( $0: 59)$}

Le messager entend un bruit indiquant que des bandits tentent de pénétrer dans le fourgon postal et verrouille la boîte contenant les objets de valeur; le messager est tué lors d'une bataille à coups de fusils; les bandits ouvrent la boîte avec de la dynamite et volent son contenu avant de quitter le wagon.

Plan 4: The Fight on the Tender $(0: 49)$

Deux bandits se hissent sur le wagon derrière la locomotive pour maîtriser l'ingénieur et le pompier; un des bandits réussit à assommer le pompier et à le jeter en bas du train alors que l'autre donne l'ordre à l'ingénieur d'arrêter le train.

\section{Plan 5: The Train Uncoupled $(0: 21)$}

Le train s'arrête et les bandits suivent l'ingénieur pendant qu'il détache la locomotive du reste des wagons; il l'avance de 100 pieds.

\section{Plan 6: Exterior of Passenger Coaches $(1: 58)$}

Les bandits font sortir les passagers des wagons, les volent, tirent à bout portant un homme essayant de s'enfuir, puis tirent en l'air pour faire peur aux autres avant de se sauver; les passagers viennent alors en aide au blessé. 
Plan 7: The Escape (0:24)

Les bandits se sauvent avec leur butin dans la locomotive qui repart.

Plan 8: Off to the Mountains $(1: 26)$

Les bandits arrêtent la locomotive et la quittent pour se sauver en descendant une colline.

\section{Plan 9: A Beautiful Scene in a Valley $(0: 51)$}

Les bandits descendent une colline, traversent un ruisseau, montent les chevaux qui les attendaient, puis se sauvent.

Plan 10 : Interior of Telegraph Office $(0: 51)$

Le télégraphiste ligoté parvient à se relever, appelle à l'aide en manipulant son télégraphe avec son menton, puis s'évanouit; en entrant dans le bureau, sa fille le découvre, le détache et lui lance de l'eau au visage pour le réveiller.

\section{Plan 11 : Interior of Dance Hall (1:08)}

Des hommes et des femmes dansent jusqu'à ce qu'un "Tenderfoot " soit reconnu et forcé de danser pendant que des hommes déchargent leurs pistolets en direction de ses pieds; le télégraphiste arrive et raconte ce qui lui est arrivé; les hommes quittent rapidement le saloon, fusils en main.

Plan 12: The Posse in Pursuit $(0: 38)$

Les bandits sont poursuivis par les justiciers et chacun décharge son fusil; un des bandits est tué.

\section{Plan 13: Battle to the Death $(1: 41)$}

Les trois autres bandits, croyant avoir semé leurs poursuivants, descendent de leurs chevaux pour compter leur butin; les justiciers arrivent sans bruit par-derrière et les attaquent: tous les bandits sont tués et le butin volé recouvert.

Plan 14: Realism (0:05)

Barnes, le chef des bandits, tire à bout portant sur chacun des membres de l'auditoire. 


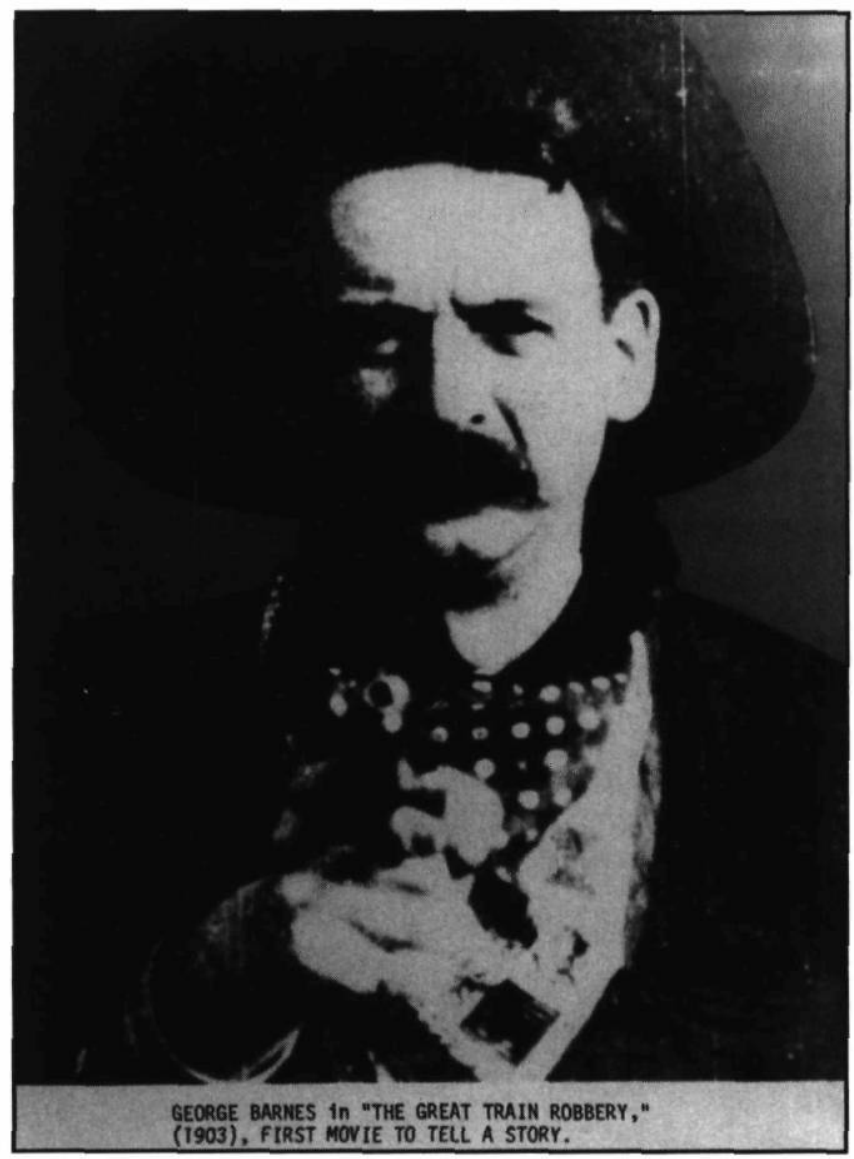

\title{
Georges Barnes dans The Great Train Robbery (1903)
}

\author{
Collection Cinémathèque québécoise
}

\section{NOTES}

1 L'essentiel de cet article a été présenté sous la forme d'une communication au congrès annuel de l'ACFAS (Association canadienne-française pour l'avancement des sciences), qui avait lieu à Rimouski du 17 au 21 mai 1993.

2 Cette classification est développée par André Gaudreault dans $D u$ littéraire au filmique. Système du récit (Québec/Paris : P.U.L./Méridiens-Klincksieck, 1988), notamment aux pages 19 et 20.

3 André Gaudreault cite et traduit dans "Théâtralité et narrativité de Georges Méliès ", Méliès et la naissance du spectacle cinématographique (Paris: Klincksieck, $1984 b$, p. 214) cette définition de Noël Burch du "mode de représentation institutionnel : : "[...] ensemble des directives (écrites ou non) qui, historiquement, ont été intériorisées par les cinéastes et les techniciens comme la base irréductible du "langage 
cinématographique" au sein de l'Institution et qui sont demeurées constantes tout au long des cinquante années [sic], indépendamment des importantes transformations stylistiques qui ont pu intervenir."

4 C'est-à-dire : raconter tout en montrant, montrer tout en racontant.

5 Nous employons le concept "Idée " au sens kantien de la forme maximisée.

6 Notre traduction de la description du Great Train Robbery présentée dans le supplément 200 du catalogue Edison (janvier 1904), reproduit par George C. Pratt à la page 34 de son ouvrage Spellbound in Darkness: Reading in the History and Criticism of the Silent Film (New York: Graphic Society, 1973).

7 Il s'agit " [...] de modes de représentation et de substances narratives ou gestuelles venues du mélodrame, du vaudeville, de la pantomime [...], de l'illusionnisme, du music-hall et du cirque; de la caricature, des images d'Épinal et autres bandes dessinées; des conteurs de rue, des numéros de foire et de music-hall, des musées de cire” ("Porter ou l'ambivalence", Le Cinéma américain: analyses de films, Paris: Flammarion, 1980 , p. 33).

8 L'on s'entend pour nommer "encyclopédie d'une époque" ce qui permet aux individus d'effectuer un travail de compréhension et d'interprétation d'énoncés divers, qu'ils soient médiatisés ou non (dans des journaux ou des affiches, au théâtre, au music-hall ou au cinéma, etc.). Pour Umberto Eco l'encyclopédie se définit comme " [...] l'ensemble enregistré de toutes les interprétations, concevable objectivement comme la bibliothèque des bibliothèques, quand bibliothèque veut dire aussi les archives de toute l'information non verbale enregistrée d'une manière ou d'une autre, des peintures rupestres aux cinémathèques" (Sémiotique et philosophie du langage, Paris: P.U.F., 1988, p. 110).

9 George A. Smith apparaît ainsi comme le premier cinéaste à alterner dans ses films des plans éloignés ou moyens et des plans rapprochés (Grandma's Reading Glasses, 1900; The Little Doctor, 1900; Mary Jane's Mishap, 1903) tandis que James Williamson met de l'avant quelques-uns des principes de base du découpage et du montage en continuité (Attack on a China Mission, 1902), que perfectionnera Frank Mottershaw dans son Attaque d'une diligence au siecle dernier (1903).

10 Pour André Gaudreault, pendant ses vingt premières années d'existence, le cinéma se présente d'ailleurs comme " [...] le lieu d'une tension entre deux régimes divergents de consommation filmique (chacun d'eux étant bien sûr lié à un régime de production spécifique) : celui qui s'établit sur un rapport de "confrontation exhibitionniste" entre l'écran et le spectateur et celui qui présuppose, tout au contraire, une "absorption diégétique" de celui-ci par l'intermédiaire de celui-là " (Du littéraire au filmique. Système du récit, Québec/ Paris: P.U.L. / Méridiens-Klincksieck, 1988, p. 25).

11 C'est par exemple le cas de L'Arrivée d'un train en gare de la Ciotat et de L'Arroseur arrosé de Louis Lumière, en 1895.

12 On pense évidemment au Voyage dans la lune de Georges Méliès (1902).

13 Pour William Everson et George Fenin, le film est en quelque sorte « [...] a blueprint for all westerns" (The Western: From Silents to Seventies, New York: Grossman, 1973, p. 49).

14 Traduction d'expressions utilisées dans le catalogue Edison, cité par Pratt dans Spellbound in Darkness: Reading in the History and Criticism of the Silent Film (New York: Graphic Society, 1973, p. 35).

15 Le mode de représentation primitif prévaut dans le régime des attractions. Voir à ce sujet De L'Arrivée d'un train à The Lonedale Operator: une trajectoire à parcourir (Montréal: Centre de documentation de la Cinémathèque, 1983).

16 Au plan 4, deux bandits se hissent sur le wagon derrière la locomotive pour mâ̂triser l'ingénieur et le chauffeur. Un des bandits réussit à assommer le chauffeur et à le 
jeter en bas du train en mouvement (la scène est spectaculaire, car le train roule à plus de $40 \mathrm{~km}$ à l'heure), tandis que l'autre ordonne à l'ingénieur d'arrêter le train. Juchée sur le wagon postal, la caméra cadre la scène de manière à faire voir la moitié supérieure du wagon où se déroule l'action (sur le tender) et la locomotive ainsi qu'une partie du paysage qui défile à vive allure. Au plan suivant, le spectateur voit le train qui arrête et les bandits qui suivent l'ingénieur pendant qu'il détache la locomotive du reste des wagons. La locomotive avance ensuite d'une centaine de pieds, ce que montre une caméra cadrant la partie inférieure gauche de l'avant du train. Au plan 6, les bandits font sortir les passagers des wagons, les volent, tirent à bout portant sur un passager qui tentait de s'enfuir (autre moment attractif), puis tirent quelques coups de feu dans les airs pour apeurer les autres avant de se sauver. Les passagers viennent alors en aide au blessé. Lors de ce plan, la caméra offre une vue frontale et centrale du train et des passagers. Au plan 7, le spectateur retrouve un cadrage semblable à celui du plan 5 et voit les bandits se sauver avec leur butin dans la locomotive.

17 Quoique nous devrions plutôt parler de "quasi-contiguïté " des espaces du plan 4 et du plan 5, car leur contiguïté ne s'embarrasse pas du fait que les personnages descendent du côté droit dans le plan 4 et qu'ils mettent pied à terre à gauche de la locomotive au plan suivant.

18 The Great Train Robbery présente le récit d'un vol de train dans ses neuf premiers plans, ce qui débute par le ligotage du télégraphiste (plan 1) et se termine par le départ des bandits, qui se sauvent à cheval avec leur butin sous le bras (plan 9). Aux plans 10 et 11 , Porter introduit une nouvelle ligne d'action au cours de laquelle nous assistons à la découverte du télégraphiste par sa fille et à sa démarche auprès des justiciers en fête dans un saloon pour que soit entreprise la poursuite des bandits. Le plan 12 montre les bandits à cheval poursuivis par les justiciers. Grâce à ce plan, le spectateur infere que les deux plans précédents constituaient un retour en arrière, de telle sorte que les plans 10 et 11 sont simultanés aux plans 1 à 9 . Car même si tout se déroule à l'intérieur d'un même syntagme qui défile dans la durée réelle et qui fait suivre une action qui a eu lieu auparavant, le spectateur comprend une bifurcation du récit, sinon il est impossible que les justiciers aient eu le temps de rattraper les bandits au plan 12 .

19 Le spectateur d'aujourd'hui ne peut, quant à lui, parvenir à comprendre The Great Train Robbery sans l'aide de ce dernier, car les règles de montage du MRI ne sont pas en vigueur à l'époque. Il serait d'ailleurs malhonnête d'évaluer le film selon de pareilles conventions.

20 Confronté au problème de devoir fonctionner avec deux lignes d'action simultanées, Porter choisit des solutions issues du système attractif: effectuer un montage successif de scènes simultanées (les plans 1 à 9 et 10-11) et confier l'explication du récit à un bonimenteur. Il se conforme donc au régime des attractions à certains endroits de son film, pour y échapper à d'autres.

21 Comme le précise Eco: "La vie de la culture est la vie de textes gouvernés par des règles intertextuelles où tout "déjà-dit" agit comme règle possible. Le déjà-dit constitue le trésor de l'encyclopédie" (Sémiotique et philosophie du langage, Paris: P.U.F., 1988, p. 273).

22 Les titres sont ceux du catalogue Edison.

\section{BIBLIOGRAPHIE}

Abel, Richard. "The "Blank Screen" of Reception", "Early French Cinema ". Iris, vol. $6, n^{\circ} 2$ (1990), p. 27-47. 
Andrew, Dudley. "Histoire esthétique et histoire culturelle». Histoire du cinéma : nouvelles approches (collectif). Paris: Publications de la Sorbonne (1989), p. 89-100.

Aumont, Jacques. "Le point de vue». Communications, $n^{\circ} 38$ (1983), p. 3-29.

Austin, John Langshaw. Quand dire c'est faire. Paris: Seuil, 1970.

Burch, Noël. "Porter ou l'ambivalence». Dans Raymond Bellour (direction), Le Cinéma américain: analyses de films, vol. 1. Paris: Flammarion (1980), p. 30-49.

Casetti, Francesco. «Le texte du film ». La théorie du film (collectif). Paris: Albatros (1980), p. 41-65.

Casetti, Francesco. "Looking for the Spectator ". Iris, vol. 1, n 2 (1984), p. 15-29.

Crafton, Donald. "Audienceship in Early Cinema ". Iris, vol. 6, n 2 (1990), p. 1-12.

Dagrada, E. "Through the Keyhole: Spectators and Matte Shots in Early Cinema ". Iris, $\mathrm{n}^{\circ} 11$ (1990), p. 95-106

Eco, Umberto. "Notes sur la sémiotique de la réception ". Actes sémiotiques, vol. IX, $n^{\circ} 81,1987$.

Eco Umberto. Sémiotique et philosophie du langage. Paris: P.U.F., 1988.

Everson, William K. et Fenin, George N. The Western: From Silents to Seventies. New York: Grossman, 1973.

Gardies, André. "L'espace du récit filmique: propositions". Cinémas de la modernité: films, theories (collectif). Paris: Klincksieck (1981), p. 75-92.

Gaudreault, André. "Temporalité et narrativité: le cinéma des premiers temps (18951908) ". Etudes littéraires, vol. 13, n 1(1980), p. 109-137.

Gaudreault, André. De L'Arrivée d'un train à The Lonedale Operator : une trajectoire à parcourir. Montréal: Centre de documentation cinématographique de la Cinémathèque québécoise, 1983.

Gaudreault, André. "Film, récit, narration : le cinéma des frères Lumière ". Iris, vol. 2, $\mathrm{n}^{\circ} 1$ (1984a), p. 61-70.

Gaudreault, André. " "Théâtralité" et "narrativité" de Georges Méliès ". Méliès et la naissance du spectacle cinématographique. Paris: Klincksieck (1984b), p. 199-219.

Gaudreault, André. Du littéraire au filmique. Système du récit. Québec/Paris: P.U.L. / Méridiens-Klincksieck, 1988.

Gaudreault, André et Gunning, Tom. "Le cinéma des premiers temps: un défi à l'histoire du cinéma ". Dans Jacques Aumont (direction), Histoire du cinéma. Nouvelles approches. Paris: Publications de la Sorbonne (1989), p. 49-63.

Gaudreault, André et Jost, François. Cinéma et récit II. Le Récit cinématographique. Paris: Nathan, 1990.

Genette, Gérard. Palimpsestes. Paris: Seuil, 1982.

Gunning, Tom. "Non-Continuity, Continuity, Discontinuity: A Theory of Genres in Early Film ". Iris, vol. 2, n 1(1984), p. 101-112.

Jost, François. "Le film comme œuvre». Protée, vol. 19, n 3 (1991), p. 9-16.

Keil, Charles. «The Story of Uncle Josh Told: Spectatorship and Apparatus in Early Cinema ". Iris, n 11 (1990), p. 63-76.

Kirby, Lynne. "The Urban Spectator and the Crowd in Early American Train Films". Iris, $\mathrm{n}^{\circ} 11$ (1990), p. 49-62.

Kuhns, William. Movies in America. Dayton: Pflaum/Standard, 1972.

Musser, Charles John. Before the Nickelodeon: Edwin S. Porter and the Edison Manufacturing Company (thèse de doctorat). New York: New York University, 1986.

Levy, David. Edwin S. Porter and the Origins of the American Narrative Film, 18941907 (thèse de doctorat). Montréal : Université McGill, 1983. 
Morin, Edgar. Le Cinéma ou l'homme imaginaire. Essai d'anthropologie sociologique. Paris : Minuit, 1956.

Odin, Roger. "Pour une sémio-pragmatique du cinéma ". Iris, vol. 1, n 1 (1983), p. 67-83.

Ottawa Film Society. The Western. Ottawa: Ottawa Film Society, 1964.

Pratt, George C. Spellbound in Darkness: Readings in the History of Criticism of the Silent Film. New York: Graphic Society Ltd., 1973.

Ricœur, Paul. "La fonction herméneutique de la distanciation ". Du texte à l'action. Paris: Seuil (1986a), p. 101-117.

Ricœur, Paul. "Qu'est-ce qu'un texte?». Du texte d̀ l'action. Paris: Seuil (1986b), p. 137-159.

Rieupeyrout, Jean-Louis. La Grande Aventure du western: du Far West dे Hollywood (1894-1964). Paris: Éditions du Cerf, 1964.

Sadoul, Georges. Histoire générale du cinéma tome 2. Les Pionniers du cinéma 18971909. Paris: Denoël, 1948.

Toulet, Emmanuelle. Cinématographe, invention du siècle. Paris: Gallimard/Réunion des musées nationaux, 1988.

Vernet, Marc. "Structure de l'espace». Dans J. Collet et al., Lectures du film. Paris: Albatros (1980), p. 86-95. 